masses have already been dealt with under projecting tonsils. I have found that the posterior mass is especially liable to become enlarged when a tonsil, the subject of enlargement in its other parts, has been removed by the guillotine during the early years of life, while the tonsil is still in a state of activity.

The tonsul with marked lingual prolongation.-The lingual prolongation may take part in a general tonsillar enlargement or may be enlarged when the rest of the tonsil is not conspicuous.

With regard to the anatomy of the tonsils in relation to the treatment of their pathological conditions the following points are of special importance. The supratonsillar fossa may form a resting place for a foreign body or calcareous particles, such as are found in the crypts. Feelings of discomfort on swallowing and pricking sensations are then noticed by the patient. If the plica semilunaris is retracted by a bent probe the foreign body can be seen and dislodged. In one case of mine a portion of fish bone was imbedded in the wall of the fossa and was only dislodged when the plica semilunaris was pulled aside. In a paper by Paterson the connexion between the fossa and peritonsillar abscess was clearly demonstrated. The supratonsillar fossa forms a convenient nidus for bacteria and pus may sometimes be squeezed out of it. It can easily be seen that should there be pus whose free outlet from the fossa is prevented because the opening is occluded by inflamed tonsil, the capsule which is here the only barrier between its cavity and the alveolar tissue space will give way and pus burrow in the pericapsular space.

With regard to the bearing which their anatomical varieties have on the technique of the successful removal of the tonsils it must be recognised: First, that the size of a tonsil is no criterion for the necessity for surgical interference. Sepsis, not size, is the determining factor. Hence it may be necessary to remove tonsils which are not enlarged. Secondly, the fact that the tonsil does not project from between the pillars is no proof that there is not a large tonsillar mass, as it may be deeply imbedded. Thirdly, that especially during the earlier years of life there seems to be a remarkable capacity of proliferation in any portion of tonsil left. With this reservation, that in a few cases there may be such proliferation after the operation, it may be said that the removal of projecting or hanging tonsils (and also in some cases of imbedded ones) by means of the guillotine is usually successful. I speak of this method when used by one thoroughly accustomed to, and much practised in, the use of the instrument.

When using the guillotine it is necessary to dislocate the tonsil with its capsule from between the pillars of the fauces and also from areolar tissue which separates it from the superior constrictor. This is accomplished by traction by a volsellum or by pressure from without by a finger on the neck and by pressure on the pillars, especially the posterior, with the ring of the guillotine; at the same time the blade is made to cut through the reflection of mucous membrane between the anterior pillar and tonsil and then to enter the areolar space outside the capsule. For this reason I prefer a blunt to a sharp blade, as the latter is more likely to cut through the deeper portion of the tonsil than to push its way between capsule and constrictor.

The plica triangularis if well developed may form an obstacle to the successful use of the guillotine, as it spreads out over the anterior portion of the tonsil and narrows the ontlet of the tonsillar space. The outlet of the tube is in this case between the free edge of the plica and the posterior pillar instead of being between the anterior and posterior pillars. The blade of the guillotine has to cut through the attachment of plica to anterior pillar before getting outside the capsule and this difficulty is increased if, as sometimes happens, the tonsillar tissue extends on to the plica triangularis. If a small portion of tonsil and capsule is left at the deepest part of the interfaucial space it usually forms a slough and disappears or else becomes fibrotic and gives no further trouble, but in some cases, as above mentioned, a renewed hypertrophy follows. Another complication which may occur is for the remains of lymphoid tissue, bound down and surrounded by fibrous bands, to continue to form a nucleus for infection. Many cases of recurrent sore throats after the operation are due to this cause. The posterior tonsillar mass is often left behind, as the guillotine is likely to cat through the capsule at this point, and it may sub. sequently hypertrophy or become inflamed. The lingual prolongation is usually left behind in removal of the tonsil by the guillotine, and $I$ have seen subsequent attacks of tonsillitis in this portion after removal of the main tonsillar mass.

In order to prevent the above sequelre it has been recommended by some authorities to curette the remains of the tonsils after the use of the guillotine by a sharp spoon or the finger ; but here again, as I have emphasised above, scraping off lymphoid tissue from the capsule without completely removing the latter does not insure success. Subsequent use of punch forceps or cautery has the same objection. I have had cases both in children and adults where these had been employed on several occasions after the use of the guillotine and in which infective lymphoid remains bound down in a mass of cicatrix caused as much trouble to the patient as the intact tonsils had done before the first operation.

The ideal method of removal is undoubtedly enucleation and this is especially the case in the imbedded tonsils of young children. In this operation the areolar tissue space outside the capsule is entered and the gland shelled out together with the lingual prolongation, with which the latter is continuous. There is then no possibility of recurrence. The bleeding is a negligible quantity if the anæsthesia is good, and in any circumstances far less than when the guillotine is used, as the vessels are severed where they enter the capsule and are thus able to retract. The condition of the throat, in children at any rate, is no worse than after other methods, nor does the healing take an appreciably longer time, while the after results are infinitely better. The use of the snare, although it effects a partial enucleation, is not so satisfactory as the complete radical operation because the lingual prolongation is not removed by this method. It is unnecessary for me'to describe the technique or details of the result of the operation, as Mr. G. E. Waugh, under whom I learnt and first practised the operation in the aural department of the Hospital for Sick Children, Great Ormond-street, has an admirable account of enucleation, with an exhaustive analysis of a large series of cases, which is now in the press.

Wimpole-street, W.

\section{THE ETIOLOGY OF THE PSORIATIC AND ALLIED CONDITIONS. ${ }^{1}$}

\section{BY MARTIN JOSEPH CHEVERS, L.R.C.S., L.R.C.P. IREL.}

WITH reference to the etiology of psoriasis there are still three theories-the "parasitic," the "light , hunger," and the "neuropathic"; which is the one we are to accept? I have treated many cases of the psoriatic condition and $I$ believe it to have a neuropathic origin, resulting in a denutrition and structural destruction of the epidermis, an exfoliation of the epidermis and dried leucocytes, brought about by a drying and shrivelling up of the epidermis for want of its necessary nutriment which it obtains by imbibition from the derma; now this condition of blood hunger is, I believe, in turn brought about by a defective general nervous system showing itself in the first instance by giving way of their weakest portion-namely, the very fime cutaneous trophic nerves and interference with the fine capillary circulation in the derma, loss of tone in these capillaries allowing of their compression by the abundant elastic fibres of the corium and the unstriped muscle fibres of its superficial layers resulting in an interrupted bloodsupply to the derma and serous exudation beneath the epidermis. In individuals whose epidermis resists rupture sufficiently long to allow of a drying up of this exudation you get exfoliated epidermis plus dry leucocytes-in other words, the psoriatic condition.

The Malpighian layer of epidermis is supplied with small nerve fibrils which are affected when the general nervous system is deficient; these delicate nerve fibres of the epidermis would with very slight disturbance be likely to become completely functionless, resulting in a drying away of the epidermis as a whole, which effect is assisted by the interposition between it and the cutis vera of the dried

1 An excerpt from an address delivered before the Manchester 1 An excerpt
Medical Society. 
exudation. Why should psoriasis occur on the skin of the dorsal surfaces and most marked on the thickest portion of these as the knee and elbow? Because the thicker the epidermis the less the number of fibres, as is evidenced by the reduced sensation in these situations, and consequently the easier to disturb the function and life of the epidermis by lessening its power of procuring its nutrition from the corium, blood-supply to the latter being curtailed by the general atonicity of the nervous system. Again, the more frequent over-stretching, owing to flexion of the limbs, of the skin of the extensor aspects tends to interfere more with the dorsal blood-supply resulting in a psoriatic condition. Now why should psoriasis be more evidenced in the cold seasons and in cold countries? This is, I think, easy to account for from the fact that to those so predisposed their nervous systems are more liable to suffer when there is a diminished sunlight combined with the disturbance and over-taxation of the skin functions by the sudden chilling and very often as sudden application of warmth to the surface rendering their fine terminal nerve endings more liable to loss of function; also can be taken into account the friction of woollen garments which act as a continual irritation to those with weak nervous systems and so the nerve terminals again suffer. Why the negro race are exempt from psoriasis can be accounted for by the fact that they spend the greater part of their lives out of doors and so possess better nervous systems, and nervous systems which are not subject to the heavy strain put upon those of the white races in their mad rush for progress. Why psoriasis does not occur in animals, being one of the few conditions found in human beings that cannot be exemplified in animals, can be explained also by their open-air life and the absence of circumstances which go to affect their nerrous systems; and to be added to these reasons may be mentioned the fact of the better tone of their nerve terminals owing to lack of irritation due to sudden variations in surface temperature by their always wearing the same thickness of clothing-their hair only; nor have they the same alterations in their dorsal skin tensions as do man. That diseases, such as syphilis and gout, are liable to bring about the condition can be explained by the effect sucti troubles are known to have on the general nervous system. The absence of the psoriatic condition from the dorsum of the foot when manifest on the dorsum of the hand can be accounted for from the fact that there is little or no disturbance of circulation in the former siluation owing to varied tension of skin; besides there is not in the case of the dorsum of the foot that thickness of epidermis with its paucity of nerve fibrils and diminished power of resistance. When psoriasis does appear in the foot it is, as you know, most frequently on the plantar aspect where the epidermis is thick; there are few nerve fibres and there is always a certain amount of disturbance of circulation from pressure. Then, again, psoriasis appears on the scalp, which can also be accounted for by its weakened circulation brought about by the sudden variations in temperature and irregular pressure caused by at one time wearing and at another time being without a head-gear, as well as a certain degree of atonicity of the nerve terminals brought about by shading off of sunlight and air by the use of covering when out of doors, the only time when full sunlight and fresh air are procurable. The tight stretching of the skin of the scalp over the bone may also be a contributory factor.

We know that psoriasis occurs frequently in miners and I think can be well attributed to the deteriorating influence the absence of sunlight and fresh air has on their nervous systems. A peculiarity about the psoriatic condition is that it is seldom seen on the face, although the face is so subject to vaso-motor disturbances by chilling of the surface and variations in temperature. Yes; but, on the other hand, the variations in tension of the skin and local pressure and friction are absent and it is not at one time covered and at another uncovered, or a lighter covering substituted for a heavier to suit the different climatic conditions. It is like the animals, always uncovered, so that the circulation in it has been trained more readily to adapt itself to variations in atmospheric and individual systemic changes. If the face does suffer, as I have seen it do, note that it picks out the very two spots that are most subject to variations in their blood-supply-namely, the malar prominences and the nose.
It is a significant fact also that psoriasis attacks the nails, which also at times suffer in nervous diseases and in neuritis I have been quite lately attending a very neurotic female who whenever she gets a nerve storm, and the arrival of which is extremely well appreciated by her husband, her nails become furrowed, long furrows reaching to their extremities, and a crack occurs along the bed of each furrow. She states that these furrows sometimes have time to grow out to the end, being gradually pared away, leaving a quite smooth nail, before she gets another round which again furrows and cracks them. During these seizures she also gets her finger ends and the plantar aspect of her feet cracked, in fact, a typical eczema rimosa; she was in this condition during my last attendance. There were also hyperæsthetic and pruritic areas on the skin of her body, fleeting from one position to another. We also know that psoriasis proper does not occur in the mucous membranes, which fact can be attributed to the nerve endings therein being stronger and retaining their medulla.

The symmetry of a psoriatic eruption points towards an abnormal condition of the general system; and that in some cases the psoriasis disappears spontaneously points in the same direction. Now I contend that angio-neurotic oedema is only a different manifestation of a like condition and one in which there is moist exudation beneath the cutis vera, moist instead of dry being accounted for owing to the locality which the manifestation chooses to adopt, as it sometimes does in the less severe types of the complaint when it chooses spots where there is loose areolar tissue and the lax texture of the latter permits of exudation taking place sooner than it otherwise would and before reaching the epidermis. I also contend that pemphigus is a similar condition, or, rather, one with a similar etiology, but in its case the exudation happens to take place beneath a resistant epidermis in contrast to a non-resistant epidermis as in eczema, and in those whose capillary walls are very permeable and who probably have a low blood coagulability. It must be remembered that there is a congenital difference in the permeability of the capillary walls and in the degree of coagulability of the blood, therefore the transudation of liquor sanguinis is more easily accomplished in some than in others and also varies in quantity ; which facts, together with the variability in the skin elasticity of individuals, may go towards explaining why in the one individual there is the dry scaly variety, psoriasis, and in the other the moist exudative variety, eczema and pemphigus. To sum up the reasons why you have eczema in the one and psoriasis in the other, when I suppose them both to have the same etiology, depends on, first, the locality which the manifestation chooses to adopt, the lax flexor or the more tense extensor aspect; secondly, the individual peculiarity with respect to the permeability of the capillary walls, the degree of coagulability of the blood and the variability of skin elasticity; and it must be remembered that you can have either of these conditions present in the same individual at the same time.

That light rays and natural sun rays do in some cases remove psoriasis is a fact and one which in itself points towards a nervous etiology.

My attention has been drawn to the report on the International Dermatological Congress held at New York in September, 1907, in which Dr. Buckley of New York is reported as stating in his paper on "The Value of an Absolutely Vegetarian Diet in Psoriasis" that many facts point to psoriasis not being a purely local disease of the skin, although the character of the individual lesions strongly suggest a parasitic element in their production. Dr. H. Radcliffe Crocker at the same Congress said he did not consider the influence of diet to have such an effect on the condition as did. an excessive use of alcohol, which statement clearly points towards a neuropathic theory. Dr. Neuberger also speaking emphasised the importance of trauma as an etiological factor. How can trauma have such an action if not through the nervous system?

Withington, Manchester.

The Cardiff Infirmary.-A sum of $£ 3250$ has been guaranteed up to the present towards an estimated needed total of $£ 5000$ for the Welsh pageant at Cardiff. Lord Aberdare has promised $£ 100$ conditionally upon any surplus being given to the Cardiff Infirmary. 